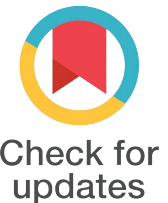

DOI: $10.21859 / \mathrm{ijcp}-03033$

\section{Left Ventricular Torsion Deformation in Atrial Septal Defect Patients Undergoing Transcatheter Device Closure}

\author{
Abdul Razak ${ }^{1, *}$, Maryam Said Mohamed Al Hajri ${ }^{1}$, K Ranjan \\ Shetty ${ }^{1}$, Krishnananda Nayak ${ }^{1}$
}

${ }^{1}$ Department of Cardiology Kasturba medical college Manipal, India

*Corresponding author: Abdul Razak U K, MD, DM, Department of Cardiology, Kasturba Medical College, Manipal, India. Tel.: +91-9448252641, E-mail: razakkaup@gmail.com

\begin{abstract}
Introduction: Atrial septal defects (ASD) considered being one of the known congenital heart diseases. ASD causes increased volume overload of the right heart. The purpose of this study was to evaluate left ventricular (LV) torsion deformation in ASD patients undergoing transcatheter device closure.

Methods: All adult patients who underwent transcatheter device closure with ostium secundum ASD were included in the study. We assessed LV torsion in ostium secundum ASD patient's pre and post device closure by using speckle tracking echocardiography.

Results: A total of 37 patients ( 22 females and 15 males) were included in this study. The average age was $28 \pm 19$ years. $L V$ peak basal rotation improved significantly $(\mathrm{P}=$ $0.028)$ in post transcatheter closure. LV torsion $\left(2.88 \pm 0.99^{\circ} / \mathrm{cm}\right.$ before vs. $3.40 \pm$ $1.41^{\circ} / \mathrm{cm}$ after closure, $\left.\mathrm{P}=0.009\right)$ and twisting $\left(15.12 \pm 4.69^{\circ}\right.$ before vs. $17.95 \pm 6.21^{\circ}$ after closure, $\mathrm{P}=0.005$ ) were statistically significant in post transcatheter closure. Volumetric assessment of LV including end-diastolic volume and systolic volume showed significant improvement $(\mathrm{P}=0.02, \mathrm{P}<0.01)$ post device closure.

Conclusions: The increased peak LV twisting and torsion was mainly attributed to the improved peak systolic clockwise rotation after ASD device closure. The LV twisting at a younger age was improved after the closure of ASD.
\end{abstract}

\section{INTRODUCTION}

Atrial septal defect (ASD) is one type of the known congenital heart diseases which account for around 30\% of adult patients and $10 \%$ of children [1-6]. While a majority of adults present symptoms such as exercise intolerance, fatigue, dyspnea, or palpitations, most of the children are asymptomatic [7]. The indication for ASD closure has been shown as a significant result in a decrease in symptoms and improvement of exercise [8, 9]. First introduced by Mills and King [10] in 1976, ASD has transitioned from surgical techniques to transcatheter base approaches using a variety of occlusion devices [11]. Amplatzer ${ }^{\circledast}$ septal occluder (St. Jude Medical, St. Paul, MN, USA) is the most commonly used device for ASD closure [10]. It is the first and only device approved by the U.S. Food and Drug Administration for clinical use of ASD closer [12].
Previous studies have demonstrated that left-to-right shunt results in an increase of right ventricular (RV) volume which may lead to increased morbidity and mortality [13-15]. Several studies show patients with $\mathrm{RV}$ and left ventricular (LV) size enhancement by echocardiographic evaluation within 24 hours undergoing percutaneous closure and normalized of biventricular size in one year $[8,9,16-18]$. At present, clinical research mechanics is moving from short and long axis methods LV function and ejection fraction to 3-dimensional ventricular deformation studies [19], including with $\mathrm{LV}$ torsion which plays an essential role for both LV systolic and diastolic function [20].

The recent two-dimensional (2D) echocardiography with the application of speckle tracking imaging (STI) has permitted estimation of LV torsional deformation 
noninvasively in comparison with sonomicrometry and magnetic resonance imaging [21, 22]. This novel technique has been demonstrated to be an accurate assessment of regional myocardial function compared with Doppler tissue imaging [23]. Torsional deformation is measured by LV angular movement about its long-axis during isovolumic contraction. Also, torsion measures the angle difference between normal base rotation in a clockwise direction and apex rotation in a counterclockwise direction [24, 25]. Additionally, studies have mainly focused on the use of Echocardiographic parameters like tissue annular velocities, strain and strain rate [26]. These parameters are also valuable for assessing LV myocardial deformation. Therefore, the present study was to investigate LV torsion deformation in ASD patients undergoing transcatheter device closure.

\section{METHODS}

The study was undertaken at a tertiary care hospital from February 2016 to February 2017. A total of 37 patients who underwent transcatheter device closure with ostium secundum ASD were included in the study. ASD associated with other congenital heart diseases, left heart valvular disease (significant stenosis or regurgitation), ejection fraction $(<55 \%)$, ischemic heart disease, hypertension, and cardiomyopathy were excluded. Before the initiation of the study, the protocol was approved by the institutional ethical committee (IEC).

\section{Percutaneous Device Closure Procedure}

The standard percutaneous device closure procedure was used. Amplatzer Septal Occluder delivered using a 7-8 Fr short sheath the right femoral vein is accessed. An arterial monitoring line can be inserted into the right femoral artery, particularly if the patients condition at the edge or procedure was performed general endotracheal anesthesia and under transesophageal echocardiography (TEE). If the femoral venous route is not available, the transhepatic approach may be advocated. The internal jugular venous or subclavian approach makes it very difficult to manoeuvre the device deployment, particularly with large defects. Administration of heparin was used to attain an active coagulation time of more than 200 seconds. Antibiotic coverage for the procedure was recommended. Commonly suggested as the use of cefazolin $1 \mathrm{~g}$ intravenously, the first dose at the time of the procedure and two subsequent doses 6-8 hours apart. Catheterization of the right heart should be performed routinely in all cases to assess the pulmonary vascular resistance. The left to right shunt calculated and a careful evaluation was also performed, considering at all aspects of the ASD anatomy (location, size, adequacy of the various rims and presence of additional defects).

\section{Patient Population and Study Design}

Detailed history, clinical parameters and cardiovascular symptoms were assessed. Echocardiography and Tissue Doppler imaging were also performed. Torsion was evaluated with STI echocardiography.

\section{Echocardiography}

Individuals were subjected for transthoracic echocardiography using Vivid 7-echocardiography system (GE) with a $2.5 \mathrm{MHz}$ transducer. Echocardiographic parameters were evaluated. The echocardiographic study was a routine evaluation done pre and post ASD device closure. The transthoracic echocardiographic study was recorded one day prior to ASD device closure (Fig.1 A and B). Early after the procedure (usually after 1 day) post echocardiographic study was done.

\section{Torsion}

LV Torsion or twisting refers to the rotational degree of a base to an apex during LV contraction. The LV torsion and twist, expressed in degrees, expressed in degrees $/ \mathrm{cm}$, both refer to the same phenomenon in the cardiac function and define the base-to-apex gradient in a rotational angle along the longitudinal axis of the LV. Torsion was assessed with speckle tracking echocardiography which allows the frame to frame track (Fig. $1 \mathrm{C}$ and D).

\section{Tissue Doppler Imaging (TDI)}

Recordings were stored digitally as two dimensional (2D) cine loops and transferred to an optical disk medium workstation for off-line analysis. The images showing the tissue motion velocity were superimposed on the $2 \mathrm{D}$ echocardiographic images for real-time colour display. TDI annular velocities during systole, early relaxation $(\mathrm{Ea})$, and atrial systole (Aa) have been possessed from LV lateral, anteroseptal and inferior wall, and an interventricular septum at the basal site in the apical 4 chamber, apical 3 chamber and apical 2 chamber views (Fig.1 E).

\section{Statistical Analysis}

Statistical analysis was carried out using Microsoft Excel spreadsheet (version 2007, Microsoft Corp, Seattle, Washington). Values were expressed as a mean \pm standard deviation or percentages. The analysis was performed by using the paired sample t-test test using SPSS software. A p value of less than 0.05 was considered as statistically significant.

\section{RESULTS}

The mean age of patients was $28 \pm 19$ years as shown in Table 1. Among 37 patients, 22 (34 \pm 19 years) were females. The first age group ( $<18$ years) has shown a clinically significant increase of LV twisting $(\mathrm{P}<0.005)$ and torsion $(\mathrm{P}=0.008)$. Similarly, in the second age group (18-40 years) $\mathrm{p}$-value was significant $(\mathrm{P}=0.043$, $\mathrm{P}=0.044)$ for twisting and torsion respectively. 

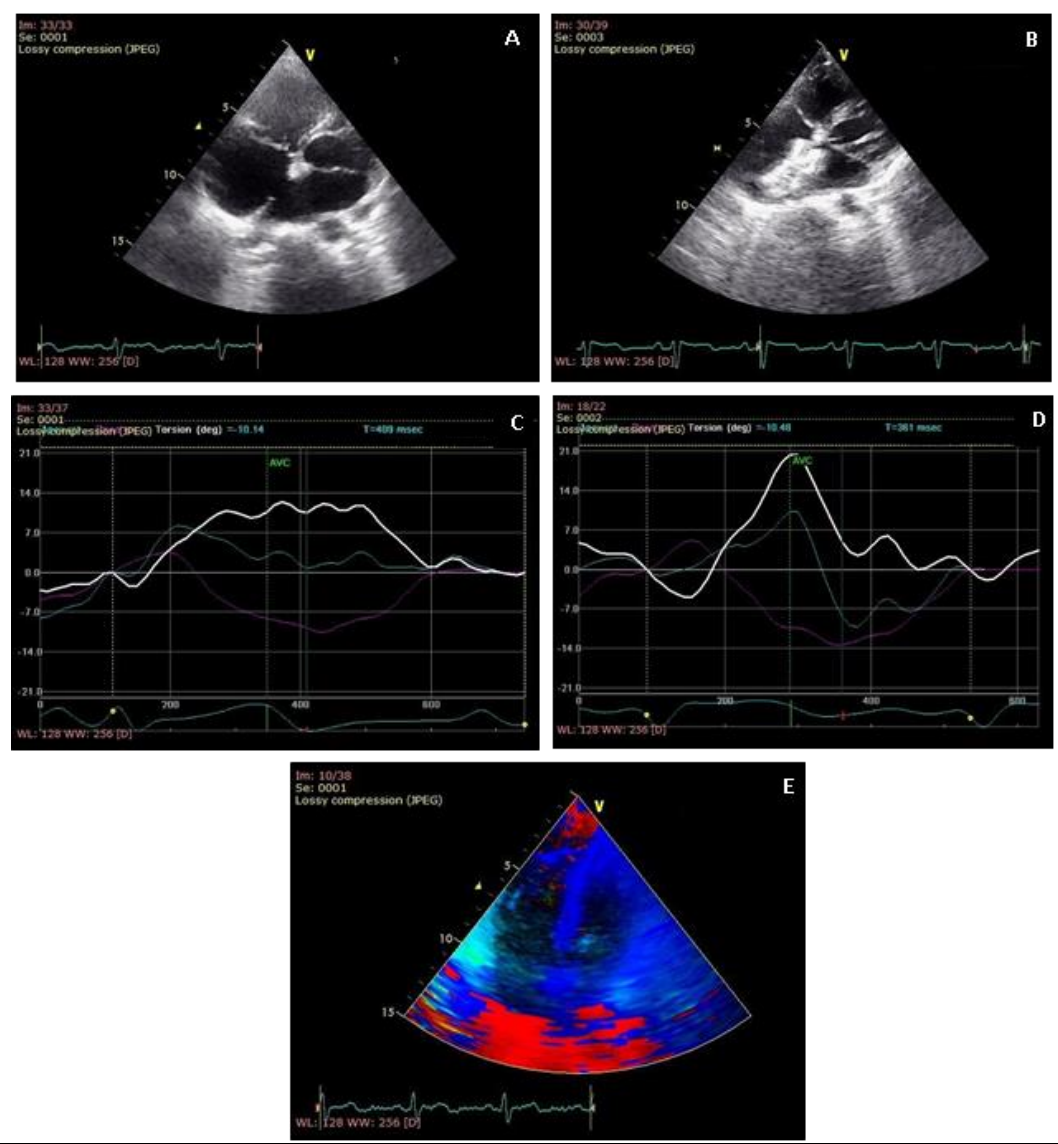

Figure 1: Transthoracic Echocardiographic Data One Day Prior to ASD Device Closure A. 2DE apical 4 chambers showing ostium secundum ASD. B. 2DE apical 4 chambers show the atrial septal device- post transcatheter closure. C. Profiles of apical rotation (blue curve), basal rotation (purple curve) and LV twist (white curve) in 5 years-old boy (OS ASD). D. Profiles of Apical rotation (blue curve), basal rotation (purple curve) and LV twist (white curve) in a 5 years- old boy (transcatheter ASD device closure). E. Apical 4 chambers view showing color tissue velocity imaging. ASD: Arterial septal defect, 2DE - 2 dimension echocardiographic; LV twist: Left ventricular twist, OS ASD: Ostium secundum Arterial septal defect

Table 1: Torsion parameters on different age groups.

\begin{tabular}{|cccc}
\hline Age groups & Pre ASD closure & Post ASD closure & P value \\
\hline$<\mathbf{1 8}$ years & & & 0.005 \\
\hline Twisting & $14.41 \pm 5.26$ & $18.75 \pm 6.50$ & 0.008 \\
\hline Torsion & $3.09 \pm 1.26$ & $3.98 \pm 1.70$ & 0.043 \\
\hline $\mathbf{1 8 - 4 0}$ years & & & 0.044 \\
Twisting & $15.21 \pm 4.42$ & $18.81 \pm 6.16$ & \\
Torsion & $2.64 \pm 0.66$ & $3.23 \pm 0.97$ & 0.831 \\
\hline $\mathbf{4 0}$ years & & & 0.720 \\
\hline Twisting & $16.04 \pm 4.66$ & $15.54 \pm 6.19$ & \\
\hline Torsion & $2.87 \pm 0.91$ & $2.71 \pm 1.12$ & \\
\hline
\end{tabular}

Data are presented as Mean \pm SD

On the other hand, the third age group (> 40 years) was insignificant for LV twisting and torsion. LV peak basal rotation was improved $(\mathrm{P}=0.028)$ in post transcatheter closure as shown in Table 2. Moreover, $\mathrm{LV}$ torsion $(2.88$ $\pm 0.99^{\circ} / \mathrm{cm}$ before vs. $3.40 \pm 1.41^{\circ} / \mathrm{cm}$ after closer, $\mathrm{P}=$ $0.009)$ and twisting $\left(15.12 \pm 4.69^{\circ}\right.$ before vs. $17.95 \pm$ $6.21^{\circ}$ after closer, $\left.\mathrm{P}=0.005\right)$ were increased significantly in post transcatheter closure. RV longitudinal deformation imaging was significant $(\mathrm{P}<$
0.001) reduced global RV strain in post ASD device closure. Furthermore, there was no significant $(\mathrm{P}>$ 0.05 ) difference in RV strain rate. Longitudinal LV global strain and strain rate were insignificant at post transcatheter closure. The basal and apical levels were improved significantly $(\mathrm{P}<0.001)$ in circumferential strain and strain rate after closure. The circumferential strain of LV papillary muscle level increased after transcatheter closure as shown in Table 2. 
Table 2: Torsion parameters of pre and post ASD closure in different age groups

\begin{tabular}{|c|c|c|c|}
\hline Variables & Pre ASD closure & Post ASD closure & P value \\
\hline \multicolumn{4}{|l|}{ Torsion parameters of all age groups } \\
\hline Peak basal rotation $\left({ }^{\circ}\right)$ & $6.16 \pm 3.32$ & $7.56 \pm 3.16$ & 0.028 \\
\hline Peak basal rotation rate $(\% / \mathrm{s})$ & $81.89 \pm 31.66$ & $85.86 \pm 37.61$ & 0.500 \\
\hline Peak apical rotation $\left({ }^{\circ}\right)$ & $9.02 \pm 4.41$ & $10.40 \pm 5.29$ & 0.118 \\
\hline Peak apical rotation rate $(\% / \mathrm{s})$ & $64.44 \pm 49.95$ & $44.52 \pm 28.55$ & 0.052 \\
\hline Torsion $(\% / \mathrm{cm})$ & $2.88 \pm 0.99$ & $3.40 \pm 1.41$ & 0.009 \\
\hline Twisting $\left({ }^{\circ}\right)$ & $15.12 \pm 4.69$ & $17.95 \pm 6.21$ & 0.005 \\
\hline \multicolumn{4}{|l|}{ Biventricular Longitudinal deformation } \\
\hline $\operatorname{LVSI}(\%)$ & $-22.73 \pm-5.04$ & $-21.95 \pm-4.78$ & 0.284 \\
\hline $\operatorname{LVSSR}(/ \mathrm{s})$ & $-1.73 \pm-0.37$ & $-1.79 \pm-0.44$ & 0.326 \\
\hline $\operatorname{LV} \operatorname{EDSR}(/ s)$ & $2.27 \pm 0.76$ & $2.21 \pm 0.86$ & 0.620 \\
\hline $\operatorname{LV} \operatorname{LDSR}(/ \mathrm{s})$ & $1.33 \pm 0.51$ & $1.29 \pm 0.49$ & 0.718 \\
\hline RVSI (\%) & $-23.36 \pm-9.32$ & $-16.89 \pm-8.59$ & 0.001 \\
\hline $\operatorname{RVSSR}(/ s)$ & $-1.93 \pm-0.68$ & $-1.74 \pm-0.72$ & 0.189 \\
\hline $\operatorname{RV} \operatorname{EDSR}(/ s)$ & $2.22 \pm 0.90$ & $1.77 \pm 1.26$ & 0.062 \\
\hline $\operatorname{RV} \operatorname{LDSR}(/ \mathrm{s})$ & $1.37 \pm 0.94$ & $1.29 \pm 0.75$ & 0.708 \\
\hline \multicolumn{4}{|l|}{ Variables (Basal level) } \\
\hline Circumferential strain (\%) & $-15.61 \pm-3.76$ & $-18.79 \pm-3.34$ & 0.001 \\
\hline Radial strain (\%) & $34.58 \pm 18.69$ & $35.83 \pm 16.22$ & 0.644 \\
\hline Circumferential strain rate(/s) & $-1.52 \pm-0.33$ & $-1.74 \pm-0.43$ & 0.003 \\
\hline Radial strain rate $(/ \mathrm{s})$ & $1.96 \pm 0.68$ & $2.12 \pm 0.94$ & 0.348 \\
\hline \multicolumn{4}{|l|}{ Variables (Papillary muscle level) } \\
\hline Circumferential strain (\%) & $-16.90 \pm-4.81$ & $-20.28 \pm-3.44$ & 0.002 \\
\hline Radial strain (\%) & $44.91 \pm 16.47$ & $50.04 \pm 13.25$ & 0.122 \\
\hline Circumferential strain rate(/s) & $-1.53 \pm-0.38$ & $-1.62 \pm-0.33$ & 0.122 \\
\hline Radial strain rate $(/ \mathrm{s})$ & $1.95 \pm 0.64$ & $2.07 \pm 0.62$ & 0.366 \\
\hline \multicolumn{4}{|l|}{ Variables (Apical level) } \\
\hline Circumferential strain (\%) & $-17.18 \pm-5.83$ & $-23.22 \pm-6.40$ & 0.001 \\
\hline Radial strain (\%) & $29.62 \pm 17.20$ & $29.00 \pm 15.83$ & 0.878 \\
\hline Circumferential strain rate(/s) & $-1.63 \pm-0.49$ & $-1.90 \pm-0.64$ & 0.017 \\
\hline Radial strain rate $(/ \mathrm{s})$ & $1.78 \pm 0.76$ & $1.81 \pm 0.79$ & 0.872 \\
\hline \multicolumn{4}{|l|}{ Variables (TDI) } \\
\hline Average LVE'(m/s) & $-0.13 \pm-0.03$ & $-0.11 \pm-0.03$ & 0.001 \\
\hline Average LV A' (m/s) & $-0.07 \pm-0.023$ & $-0.07 \pm-0.02$ & 0.566 \\
\hline Average LV S’ (m/s) & $0.1058 \pm 0.04$ & $0.09 \pm 0.02$ & 0.154 \\
\hline $\operatorname{RVE}^{\prime}(\mathrm{m} / \mathrm{s})$ & $-0.1670 \pm-0.05$ & $-0.12 \pm-0.04$ & 0.001 \\
\hline $\mathrm{RVA}^{\prime}(\mathrm{m} / \mathrm{s})$ & $-0.1284 \pm-0.04$ & $-0.11 \pm-0.04$ & 0.017 \\
\hline $\operatorname{RVS}(\mathrm{m} / \mathrm{s})$ & $0.1589 \pm 0.03$ & $0.14 \pm 0.04$ & 0.041 \\
\hline
\end{tabular}

Data are presented as Mean \pm SD

LV: left ventricle, RV: right ventricle, LVSI: left ventricle systolic strain imaging, SSR: Systolic strain rate, EDSR: Early diastolic strain rate, LDSR: Late diastolic strain rate, RVSI: right ventricle systolic strain imaging, TDI: Tissue doppler imaging, E’: Early diastolic tissue annular velocity, A': Late diastolic tissue annular velocity, S: Systolic annular velocity.

LV tissue annular velocity E' (early diastolic) was significantly $(\mathrm{P}<0.001)$ reduced after device closure. RV tissue annular velocities E' (early diastolic), A' (late diastolic) and $S$ (systolic) waves were reduced significantly $(\mathrm{P}<0.001, \mathrm{P}=0.017$ and $\mathrm{P}=0.041)$ following the closure, respectively. Maximum aortic cusp separation has increased significantly $(\mathrm{P}=0.002)$ after device closure as shown in Table 3. LV enddiastolic dimension and interventricular septum in systole were improved significantly $(\mathrm{P}<0.05, \mathrm{P}<0.001)$ after device closure. However, ejection fraction and fractional shortening were increased significantly $(\mathrm{P}<$
0.001) after device closure. Volumetric assessment of $\mathrm{LV}$ including end diastolic and systolic volume showed significant improvement $(\mathrm{P}=0.02, \mathrm{P}<0.01)$ post device closure. Also, tricuspid and pulmonary flow showed a significant reduction $(\mathrm{P}<0.001)$, while aortic flow was significantly improved $(\mathrm{P}<0.001)$ after device closure. Echocardiography revealed a significant reduction of RV free wall thickness $(\mathrm{P}<0.001)$, RV end-diastolic dimension ( $\mathrm{P}=0.04), \mathrm{RV}$ end-diastolic dimension in short and long axis $(\mathrm{P}=0.007, \mathrm{P}<0.001)$. Also, Tricuspid annular plane systolic excursion was reduced significantly $(\mathrm{P}<0.001)$ post device closure. $\mathrm{RV}$ 
fractional area change was significantly decreased $(\mathrm{P}<$ 0.001) after the procedure. Continuous wave Doppler of RV systolic pressure revealed a significant reduction
$(\mathrm{P}<0.001)$ compared with pre-device closure. Tricuspid valve inflow Doppler was significantly $(\mathrm{P}=$ 0.02 ) reduced to early diastolic or late diastolic ratio.

Table 3: Left and Right Ventricular Parameter Pre and Post Closure

\begin{tabular}{|c|c|c|c|}
\hline Variables & Pre ASD closure & Post ASD closure & Pvalue \\
\hline \multicolumn{4}{|l|}{ Aortic and LA m-mode dimensions } \\
\hline Aortic root $(\mathrm{mm})$ & $24.35 \pm 5.71$ & $24.18 \pm 5.52$ & 0.729 \\
\hline MACS (mm) & $15.35 \pm 2.75$ & $16.00 \pm 2.84$ & 0.002 \\
\hline LA dimension $(\mathrm{mm})$ & $29.75 \pm 7.67$ & $30.70 \pm 7.78$ & 0.038 \\
\hline \multicolumn{4}{|l|}{ LV function parameters } \\
\hline $\operatorname{IVSD}(\mathrm{cm})$ & $0.92 \pm 0.19$ & $0.94 \pm 0.15$ & 0.387 \\
\hline $\operatorname{IVSS}(\mathrm{cm})$ & $1.10 \pm 0.18$ & $1.21 \pm 0.26$ & 0.005 \\
\hline $\operatorname{LVEDD}(\mathrm{cm})$ & $3.53 \pm 0.52$ & $3.81 \pm 0.52$ & 0.001 \\
\hline $\operatorname{LVESD}(\mathrm{cm})$ & $2.28 \pm 0.42$ & $2.31 \pm 0.36$ & 0.471 \\
\hline $\operatorname{PWD}(\mathrm{cm})$ & $0.69 \pm 0.21$ & $0.67 \pm 0.20$ & 0.529 \\
\hline PWS (cm) & $1.06 \pm 0.24$ & $1.04 \pm 0.25$ & 0.598 \\
\hline $\mathrm{EF}(\%)$ & $66.37 \pm 6.30$ & $71.16 \pm 3.69$ & 0.001 \\
\hline FS (\%) & $35.94 \pm 4.67$ & $39.89 \pm 2.85$ & 0.001 \\
\hline $\operatorname{IVC}(\%)$ & $47.82 \pm 10.70$ & $50.67 \pm 7.37$ & 0.077 \\
\hline $\operatorname{EDV}(\mathrm{ml})$ & $36.54 \pm 14.98$ & $41.16 \pm 15.48$ & 0.021 \\
\hline $\operatorname{ESV}(\mathrm{ml})$ & $10.61 \pm 4.58$ & $12.37 \pm 5.61$ & 0.014 \\
\hline ALEF (\%) & $70.18 \pm 5.51$ & $70.13 \pm 5.07$ & 0.953 \\
\hline \multicolumn{4}{|l|}{ Valvular velocities } \\
\hline Mitral velocity $(\mathrm{m} / \mathrm{s})$ & $0.93 \pm 0.21$ & $0.93 \pm 0.23$ & 0.927 \\
\hline Aortic velocity (m/s) & $1.01 \pm 0.23$ & $1.15 \pm 0.22$ & 0.001 \\
\hline Tricuspid velocity $(\mathrm{m} / \mathrm{s})$ & $0.96 \pm 0.24$ & $0.67 \pm 0.16$ & 0.001 \\
\hline Pulmonary velocity $(\mathrm{m} / \mathrm{s})$ & $1.25 \pm 0.36$ & $0.97 \pm 0.36$ & 0.001 \\
\hline \multicolumn{4}{|l|}{ RV function parameters } \\
\hline $\operatorname{RVFW}(\mathrm{mm})$ & $7.67 \pm 1.68$ & $7.10 \pm 1.72$ & 0.001 \\
\hline $\operatorname{RVEDD}(\mathrm{mm})$ & $27.97 \pm 8.95$ & $25.70 \pm 7.86$ & 0.041 \\
\hline RVEDsax (mm) & $38.59 \pm 9.97$ & $35.48 \pm 8.93$ & 0.007 \\
\hline $\operatorname{RVEDlax}(\mathrm{mm})$ & $50.62 \pm 9.49$ & $45.05 \pm 8.34$ & 0.001 \\
\hline RVSP by TR (mmHg) & $34.10 \pm 13.94$ & $27.78 \pm 12.14$ & 0.001 \\
\hline RVFAC (\%) & $50.22 \pm 6.54$ & $44.65 \pm 6.06$ & 0.001 \\
\hline TAPSE (mm) & $22.91 \pm 4.21$ & $19.94 \pm 3.20$ & 0.001 \\
\hline TVE/A ratio & $1.66 \pm 0.53$ & $1.45 \pm 0.40$ & 0.020 \\
\hline $\operatorname{IVRT}(\mathrm{ms})$ & $54.02 \pm 19.02$ & $57.45 \pm 23.68$ & 0.343 \\
\hline Deceleration time (ms) & $130.54 \pm 33.88$ & $132.67 \pm 35.43$ & 0.769 \\
\hline Tie index & $0.40 \pm 0.16$ & $0.41 \pm 0.16$ & 0.814 \\
\hline
\end{tabular}

Data are presented as Mean \pm SD

MACS: Maximum aortic cusp separation, LA: Left atrium, IVSD: Inter ventricular septum in diastole, IVSS: Inter ventricular septum in systole, LVEDD: Left ventricular end diastolic dimension, LVESD: Left ventricular end systolic dimension, PWD: Posterior wall in diastole, PWS: Posterior wall in systole, EF: Ejection fraction, FS: Fractional shortening, IVC: Inferior vena cava, EDV: End diastolic volume, ESV: End systolic volume, ALEF: Area length ejection fraction, RVFW: Right ventricular free wall, RVEDD: Right ventricular end diastolic dimension, RVEDsax: Right ventricular end diastolic dimension in short axis, RVEDlax: Right ventricular end diastolic dimension in long axis, RVSP by TR: Right ventricular systolic pressure by tricuspid regurgitation velocity, RVFAC: Right ventricular fractional area change, TAPSE: Tricuspid annular plane systolic excursion, TV-E: Tricuspid valve Early diastolic velocity, TV-A: Tricuspid valve late diastolic velocity, IVRT: Iso-volemic relaxation time.

\section{DISCUSSIONS}

The present study elucidated LV torsional deformation in ASD patients pre and post transcatheter device closure. The transcatheter device closure increased twisting degree and wringing motion of the LV at basal and apical levels by using the STI method. The LV torsion has improved immediately after device closure based on loading conditions rather than ventricular remodeling. The peak basal rotational shown a significant improvement compared to the unchanged apical rotation. In similar findings by Dong et al., LV basal rotation was improved in their patients with ASD compared to the unchanged apical rotation [27]. LV torsion indicated the difference between the rotation of the base and apex relative to its long axis. The change in circumferential strain and strain rate was significantly 
superior in post status due to the basal rotation and used as an indicator of LV response to normalized loading conditions.

This was mainly contributed to the reduction effect of ASD volume and pressure overload over LV twisting. Cakal et al. [28] demonstrated left atrium, LV strain and strain rate did not change significantly in ASD patients. However, correction of the long-standing volume overload by percutaneous closure caused an early increase in LA and LV longitudinal deformation that associates with the magnitude of the ASD. But this was either increase or decreased in one month after closure. In the present study, our findings showed that LV longitudinal strain and strain rate were not affected in the early post status which agreed with Cakal et al. [28]. Our findings showed that longitudinal strain commonly affected by the presence of myocardial disease. However, at present, the LV longitudinal strain was unchanged in ASD with abnormal loading conditions. Moreover, RV longitudinal deformation imaging significant reduction of global RV strain and maybe contribute to the reduced RV volume overload. In the present study, circumferential strain and strain rate of basal and apical levels significantly increased of basal twisting to the acute change in loading condition. Therefore, circumferential strain can be used as an indicator of $\mathrm{LV}$ response to normalized loading conditions. This statement was supported by two recent studies $[27,29]$ on LV torsional deformation in patients with ASD before and 24 hours after percutaneous closure. Bussadori et al. [29] and Dong et al. [27] demonstrated a significant increase in LV systolic twist after the procedure due to increased basal clockwise rotation while apical counter clockwise remained unchanged. Initially, ASD symbolizes a clinical model of chronic RV volume overload. The present study revealed a significant reduction of Tissue Doppler velocities of RV systolic phase ( $\mathrm{s}^{\prime}$ ), early (e') and late (a') diastolic phases. The velocity changes represented a response to altered left and right ventricular loading conditions.

Our data is supported by previous studies showing immediate significant remodeling of cardiac geometry early after the closure. Burns et al. [20] found an early decrease in RV end-diastolic volume after ASD closure with a device or by surgery. Qin et al. [30] reported a decrease in the RV end-diastolic volume during a median follow-up period of 6 months after ASD closure. Similarly, Pascotto et al. [18] demonstrated significant positive cardiac geometric remodeling early following the percutaneous closure of ASD. Additionally, Du et al. [31] reported that after transcatheter closure of ASD, $\mathrm{RV}$ volume overload decreased within 24 hours. The decline of the RV size continued so that the mean size on the four-chamber view was found within normal limits at one month and maintained at 6 and 12 months. Therefore, RV remodeling and early cardiac geometric change has been documented in the early stage following percutaneous ASD closure.

\section{Study Limitations}

This study was performed with a small number of patients, and the follow-up period was short. Further studies need to identify the incidence of various aging on LV torsion among subjects going for ASD device closure.

\section{CONCLUSION}

The assessment of left ventricular twisting and torsional deformation was practically realistic using the noninvasive speckle tracking imaging method. Left ventricular twisting and torsion was increased peak systolic clockwise rotation after atrial septal defect device closure. The left ventricular twisting at a younger age was improved after a closure of the atrial septal defect.

\section{Conflicts of Interest}

The authors declare no conflicts of interest.

\section{Author Contributions}

Design: Razak Abdul, Maryam Said Mohd. Al Hajri, K Ranjan Shetty, Krishnananda N. Data collection: Razak Abdul, Maryam Said Mohd. Al Hajri, K Ranjan Shetty, Krishnananda N. Data analysis and interpretation: Razak Abdul, Maryam Said Mohd. Al Hajri, K Ranjan Shetty, Krishnananda N Drafting the article: Razak Abdul. Final writing: Maryam Said Mohd. Al Hajri, K Ranjan Shetty, Krishnananda N

\section{REFERENCES}

1. Tanoue Y, Morita S, Ochiai Y, Masuda M, Tominaga R. Impact of atrial septal defect closure on right ventricular performance. Circ J. 2006;70(7):909-12. doi: 10.1253/circj.70.909 pmid: 16799247

2. Stellin G, Vida VL, Padalino MA, Rizzoli G, editors. Surgical outcome for congenital heart malformations in the adult age: a multicentric European study. Seminars in Thoracic and Cardiovascular Surgery: Pediatric Cardiac Surgery Annual; 2004: Elsevier.

3. Yamada T, McElderry HT, Muto M, Murakami Y, Kay GN. Pulmonary vein isolation in patients with paroxysmal atrial fibrillation after direct suture closure of congenital atrial septal defect. Circ J. 2007;71(12):1989-92. doi: 10.1253/circj.71.19 89 pmid: 18037759

4. Ogawa T, Saga T, Nakamoto S. Surgical repair for atrial septal defect associated with myotonic dystrophy. Circ J. 2007;71(8):1321-2. doi: 10.1253/circj.71.1321 pmid: 17652 903

5. Brickner ME, Hillis LD, Lange RA. Congenital heart disease in adults. First of two parts. N Engl J Med. 2000;342(4):256-63. doi: 10.1056/NEJM200001273420407 pmid: 10648769

6. Tuncer M, Ekim H, Gunes Y, Guntekin U. Atrial septal defect presenting with Brucella endocarditis. Circ J. 2008;72(12):2096-7. doi: 10.1253/circj.CJ-07-0980 pmid: 18854616

7. Gatzoulis MA, Redington AN, Somerville J, Shore DF. Should atrial septal defects in adults be closed? Ann Thorac Surg. 1996;61(2):657-9. doi: 10.1016/0003-4975(95)01043-2 pmid: 8572783

8. Kaya MG, Baykan A, Dogan A, Inanc T, Gunebakmaz O, Dogdu $\mathrm{O}$, et al. Intermediate-term effects of transcatheter 
secundum atrial septal defect closure on cardiac remodeling in children and adults. Pediatr Cardiol. 2010;31(4):474-82. doi: 10.1007/s00246-009-9623-y pmid: 20084376

9. Khan AA, Tan JL, Li W, Dimopoulos K, Spence MS, Chow P, et al. The impact of transcatheter atrial septal defect closure in the older population: a prospective study. JACC Cardiovasc Interv. 2010;3(3):276-81. doi: 10.1016/j.jcin.2009.12.011 pmid: 20298984

10. Everett $\mathrm{AD}$, Jennings J, Sibinga E, Owada C, Lim DS, Cheatham J, et al. Community use of the amplatzer atrial septal defect occluder: results of the multicenter MAGIC atrial septal defect study. Pediatr Cardiol. 2009;30(3):240-7. doi: 10.1007/s00246-008-9325-x pmid: 19015911

11. Ermis P, Franklin W, Mulukutla V, Parekh D, Ing F. Left ventricular hemodynamic changes and clinical outcomes after transcatheter atrial septal defect closure in adults. Congenit Heart Dis. 2015;10(2):E48-53. doi: 10.1111/chd.12204 pmid: 25059679

12. Du ZD, Hijazi ZM, Kleinman CS, Silverman NH, Larntz K, Amplatzer I. Comparison between transcatheter and surgical closure of secundum atrial septal defect in children and adults: results of a multicenter nonrandomized trial. J Am Coll Cardiol. 2002;39(11):1836-44. doi: 10.1016/S0735-1097 (02)01862-4 pmid: 12039500

13. Webb G, Gatzoulis MA. Atrial septal defects in the adult: recent progress and overview. Circulation. 2006;114(15):1645-53. doi: 10.1161/CIRCULATIONAHA. 105.592055 pmid: 17030704

14. de Lezo JS, Medina A, Romero M, Pan M, Segura J, Caballero E, et al. Effectiveness of percutaneous device occlusion for atrial septal defect in adult patients with pulmonary hypertension. Am Heart J. 2002;144(5):877-80. doi: 10.1067/mhj.2002.126121 pmid: 12422159

15. Van De Bruaene A, Delcroix M, Pasquet A, De Backer J, Paelinck B, Morissens M, et al. The importance of pulmonary artery pressures on late atrial arrhythmia in transcatheter and surgically closed ASD type secundum. Int J Cardiol. 2011;152(2):192-5. doi: 10.1016/j.ijcard.2010.07.014 pmid: 20674051

16. Jategaonkar S, Scholtz W, Schmidt H, Horstkotte D. Percutaneous closure of atrial septal defects: echocardiographic and functional results in patients older than 60 years. Circ Cardiovasc Interv. 2009;2(2):85-9. doi: 10.1161/CIRCINTERVENTIONS.108.814046 pmid: 20031 700

17. Mainzer G, Braver Y, Khoury A, Schwartz Y, Galenter-Yaniv L, Yalonetsky S, et al. Morphologic, mechanical, conductive, and hemodynamic changes following transcatheter closure of atrial septal defect. Congenit Heart Dis. 2010;5(1):25-31. doi: 10.1111/j.1747-0803.2009.00371.x pmid: 20136854

18. Pascotto M, Santoro G, Cerrato F, Caputo S, Bigazzi MC, Iacono $\mathrm{C}$, et al. Time-course of cardiac remodeling following transcatheter closure of atrial septal defect. Int J Cardiol. 2006;112(3):348-52. doi: 10.1016/j.ijcard.2005.10.008 pmid: 16303193

19. Buckberg GD, Weisfeldt ML, Ballester M, Beyar R, BurkhoffD, Coghlan $\mathrm{HC}$, et al. Left ventricular form and function: scientific priorities and strategic planning for development of new views of disease. Circulation. 2004;110(14):e333-6. doi: 10.1161/01.CIR.0000143625.56882.5C pmid: 15466651

20. Burns AT, La Gerche A, Prior DL, Macisaac AI. Left ventricular untwisting is an important determinant of early diastolic function. JACC Cardiovasc Imaging. 2009;2(6):709-16. doi: 10.1016/j.jcmg.2009.01.015 pmid: 19520340

21. Helle-Valle T, Crosby J, Edvardsen T, Lyseggen E, Amundsen $\mathrm{BH}$, Smith $\mathrm{HJ}$, et al. New noninvasive method for assessment of left ventricular rotation: speckle tracking echocardiography. Circulation. 2005;112(20):3149-56. doi: 10.1161/CIRCUL ATIONAHA.104.531558 pmid: 16286606

22. Notomi Y, Lysyansky P, Setser RM, Shiota T, Popovic ZB, Martin-Miklovic MG, et al. Measurement of ventricular torsion by two-dimensional ultrasound speckle tracking imaging. J Am Coll Cardiol. 2005;45(12):2034-41. doi: 10.1016/j.jacc.2005 .02 .082 pmid: 15963406

23. Di Salvo G, Pacileo G, Caso P, Verrengia M, Rea A, Santoro G, et al. Strain rate imaging is a superior method for the assessment of regional myocardial function compared with Doppler tissue imaging: a study on patients with transcatheter device closure of atrial septal defect. J Am Soc Echocardiogr. 2005;18(5):398-400. doi: 10.1016/j.echo.2004.09.009 pmid: 15891748

24. Sengupta PP, Korinek J, Belohlavek M, Narula J, Vannan MA, Jahangir A, et al. Left ventricular structure and function: basic science for cardiac imaging. J Am Coll Cardiol. 2006;48(10):1988-2001. doi: 10.1016/j.jacc.2006.08.030 pmid: 17112989

25. Delhaas T, Kotte J, van der Toorn A, Snoep G, Prinzen FW, Arts T. Increase in left ventricular torsion-to-shortening ratio in children with valvular aortic stenosis. Magn Reson Med. 2004;51(1):135-9. doi: 10.1002/mrm.10679 pmid: 14705053

26. Rahman Helmy Ali HA, Razik Mohamad NA, El-Deen Mohammad HS, Abdul Hamid SK. 2-D Speckle tracking in the assessment of left and right ventricular functions in hemodialysis versus recently diagnosed uremic patients with preserved systolic function. Egypt J Crit Care Med. 2016;4(3):139-44. doi: 10.1016/j.ejccm.2016.08.001

27. Dong L, Zhang F, Shu X, Zhou D, Guan L, Pan C, et al. Left ventricular torsional deformation in patients undergoing transcatheter closure of secundum atrial septal defect. Int J Cardiovasc Imaging. 2009;25(5):479-86. doi: 10.1007/s10554-009-9458-x pmid: 19360478

28. Cakal S, Eroglu E, Baydar O, Cakal B, Yazicioglu MV, Bulut M, et al. Two-dimensional strain and strain rate imaging of the left atrium and left ventricle in adult patients with atrial septal defects before and after the later stage of percutaneous device closure. Echocardiography. 2015;32(3):470-4. doi: 10.1111/echo. 12693 pmid: 25059711

29. Bussadori C, Oliveira P, Arcidiacono C, Saracino A, Nicolosi E, Negura D, et al. Right and left ventricular strain and strain rate in young adults before and after percutaneous atrial septal defect closure. Echocardiography. 2011;28(7):730-7. doi: 10.1111/j.1540-8175.2011.01434.x pmid: 21615485

30. Qin Y-W, Chen L, Bai Y, Wang F-Y, Zhang Z-G, Shan X-H, et al. Transcatheter Closure of Atrial Septal Defects Improves Cardiac Remodeling and Function of Adult Patients with Permanent Atrial Fibrillation. Chinese Medical Journal. 2015;128(6):780. doi: 10.4103/0366-6999.152617 pmid: PMC4833982

31. Du ZD, Cao QL, Koenig P, Heitschmidt M, Hijazi ZM. Speed of normalization of right ventricular volume overload after transcatheter closure of atrial septal defect in children and adults. Am J Cardiol. 2001;88(12):1450-3, A9. doi: 10.1016/S0002-9149(01)02135-X pmid: 11741575 